\title{
Editorial: Alcohol Consumption and Liver Diseases: From Pathology to Phytotherapy
}

\author{
Songtao $\mathrm{Li}^{1 *}$, Zhenyuan Song ${ }^{2}$, Ping $\mathrm{Yao}^{3}$ and Jiangjiang $\mathrm{Qin}^{4}$ \\ ${ }^{1}$ School of Public Health, Zhejiang Chinese Medical University, Hangzhou, China, ${ }^{2}$ Department of Kinesiology and Nutrition, \\ University of Illinois at Chicago, Chicago, IL, United States, ${ }^{3}$ School of Public Health, Tongji Medical College, Huazhong University \\ of Science \& Technology, Wuhan, China, ${ }^{4}$ The Cancer Hospital of the University of Chinese Academy of Sciences (Zhejiang \\ Cancer Hospital), Institute of Basic Medicine and Cancer (IBMC), Chinese Academy of Sciences, Hangzhou, China
}

Keywords: alcoholic liver disease, hepatic steatosis, liver injury, phytochemicals, phytotherapy

\section{Editorial on the Research Topic}

Alcohol Consumption and Liver Diseases: From Pathology to Phytotherapy

Globally, there are 2.4 billion people who consume alcohol with a high proportion of $39.5 \%$ of heavy episodic drinkers among them (GBD 2016 Alcohol Collaborators, 2018). Emerging evidence showed that harmful alcohol consumption is associated with more than 200 diseases and injuries, and leads to 3.3 million deaths worldwide annually (Seitz et al., 2018). As an important site for alcohol metabolism, the liver becomes the main target organ of chronic and heavy alcohol consumptioninduced injury, which is referred to as alcoholic liver disease (ALD). The spectrum of ALD develops from steatosis to steatohepatitis, with some individuals ultimately progressing to fibrosis, cirrhosis, and even hepatocellular carcinoma. The perniciousness of ALD has not attracted enough attention, since the incidence rate of ALD in the population is much less than that of other liver diseases, such as non-alcoholic fatty liver disease. But in fact, ALD is the main source of terminal liver disease in clinic. Alcohol is responsible for about $25 \%$ of cirrhosis deaths and $30 \%$ of all liver cancer deaths annually (Asrani et al., 2021). Considering the significance of ALD on public health, Frontiers in Pharmacology organized this Research Topic entitled "Alcohol Consumption and Liver Diseases: from Pathology to Phytotherapy" to present recent advances in this field.

\section{PATHOLOGICAL MECHANISM OF ALCOHOLIC LIVER DISEASE}

Specialty section:

This article was submitted to Gastrointestinal and Hepatic Pharmacology,

a section of the journal

Frontiers in Pharmacology

Received: 04 January 2022 Accepted: 19 January 2022 Published: 16 February 2022

Citation:

Li S, Song Z, Yao P and Qin J (2022) Editorial: Alcohol Consumption and Liver Diseases: From Pathology to Phytotherapy.

Front. Pharmacol. 13:848334 doi: 10.3389/fphar.2022.848334

During the past decades, progress has been made on the understanding of pathological mechanism(s) of ALD. Ethanol oxidation by alcohol dehydrogenase and aldehyde dehydrogenase leads to a reduction of the $\mathrm{NAD}^{+} / \mathrm{NADH}$ ratio, and then disturbs fatty acid oxidation, glycolysis and glucogenesis, and the tricarboxylic acid cycle, contributing to hepatic steatosis; meanwhile, ethanol oxidation by the microsomal ethanol oxidizing system causes excessive reactive oxygen species generation, which further attacks biological macromolecules resulting in liver injury (Ceni et al., 2014). Emerging evidence shows that alcohol-mediated modification on intracellular biological macromolecules, such as RNA, DNA, and protein, as well as epigenetic modification, contributes to the development of ALD (Smathers et al., 2011; Chen et al., 2020; Dou et al., 2020; Dou et al., 2021; Zhang et al., 2021).

Impaired intestinal flora homeostasis, disturbance of flora metabolites, and loss of intestinal barrier integrity, as well as gut-liver crosstalk have been recognized as novel contributors to ALD (Hartmann et al., 2015). Recognition of pathogens and pathogen-associated molecules is determined by germline-encoded pattern recognition receptors, including toll-like receptors (TLRs). TLR4 
activation has been reported to be implicated in the pathological process of ALD (Hritz et al., 2008), however, as another fundamental immunoreceptor, the biological function of TLR9 in ALD is still not fully illustrated. In this Research Topic, Hao et al. reported that mice with TLR9 deficiency were more susceptible to chronic alcohol-induced oxidative stress, endoplasmic reticulum (ER) stress, and liver injury; while, TLR9 activation by CpG oligodeoxynucleotides improved ethanol metabolite and acetaldehyde-induced hepatocyte injury. However, they also observed that TLR9 deficiency protected against alcohol-induced inflammation in mice liver, indicating the bidirectional regulation of TLR9 on liver damage and inflammation in ALD (Hao et al.). This study enhanced our understanding of TLR9 in ALD. Alcohol consumption is usually accompanied by food intake. Therefore, nutritional factors, such as dietary fatty acids, modulate susceptibility to ALD. Diet with high n6-polyunsaturated fatty acids (PUFAs) aggravates, while, n3-PUFAs ameliorates early steatosis in ALD (Tull et al., 2009; Patterson et al., 2012). Warner et al. established an early alcoholic hepatitis $(\mathrm{AH})$ model, and reported that enhancing endogenous n3/n6-PUFAs ratio by transgenic Fat-1 protected AH in mice. This study provided the first clue that enriching the endogenous $\mathrm{n} 3$-PUFAs level is a potential way to improve $\mathrm{AH}$.

\section{PHYTOTHERAPY OF ALCOHOLIC LIVER DISEASE}

Currently, there are no accepted therapies available to prevent or cure ALD. Alcohol abstinence is the most common and most effective therapy to attenuate and even reverse the disease. The potential mechanism(s) involved in alcohol abstinence-improved alcoholic fatty liver remain unclear. Pi et al. explored the molecular basis of alcohol withdrawal-regulated metabolism reprogramming. Their findings enhanced our understanding of the biological mechanisms of alcohol abstinence on ALD treatment. Besides alcohol abstinence, searching for effective compounds to treat ALD is another essential and urgent task to prevent the development of ALD or treat it clinically. Phytochemicals, possessing strong abilities in defending hepatic oxidative stress, endoplasmic reticulum stress, and inflammation, which are hallmarks of ALD, are potential candidates for ALD therapy. Here, in this Research Topic, Yan

\section{REFERENCES}

Asrani, S. K., Mellinger, J., Arab, J. P., and Shah, V. H. (2021). Reducing the Global Burden of Alcohol-Associated Liver Disease: A Blueprint for Action. Hepatology 73 (5), 2039-2050. doi:10.1002/hep.31583

Ceni, E., Mello, T., and Galli, A. (2014). Pathogenesis of Alcoholic Liver Disease: Role of Oxidative Metabolism. World J. Gastroenterol. 20 (47), 17756-17772. doi:10.3748/wjg.v20.i47.17756

Chen, L., Huang, W., Wang, L., Zhang, Z., Zhang, F., Zheng, S., et al. (2020). The Effects of Epigenetic Modification on the Occurrence and Progression of Liver Diseases and the Involved Mechanism. Expert Rev. Gastroenterol. Hepatol. 14 (4), 259-270. doi:10.1080/17474124.2020. 1736042 et al. summarized 23 kinds of phytochemicals with strong antiALD effects based on clinical studies or animal experiments. Via employing a more appropriate model to mimic heavy alcohol drinkers with a Western diet, Jiang et al. reported that cannabidiol, a botanical component extracted from marijuana, protected chronic ethanol plus high-fat high-cholesterol dietinduced hepatic steatosis, oxidative stress, and inflammation in ALD mice. Gao et al. reported for the first time that kinsenoside, an active ingredient extracted from A. roxburghii, possessed the robust ability to alleviate chronic alcohol feeding-induced oxidative stress, ER stress, and liver injury via stimulating AMPK-dependent autophagy. Liu et al. reported antihepatocellular carcinoma activity of a new saponin Dioscorea Zingiberensis via regulating LncRNA TCONS-00026762. Since hepatocellular carcinoma is one of most adverse outcomes in ALD, the beneficial roles of Dioscorea Zingiberensis on alcoholassociated liver cancer is worthy of further study. Additionally, accumulating clinical evidence confirmed the effectiveness of traditional Chinese medicine (TCM) in ALD treatment. Different from Western medicine, TCM is composed of many components and acts on multi-targets. In this Research Topic, we collected one TCM prescription, named Zhi Gan prescription, which was composed of six herbal medicines and containing 138 predicted active components. The authors reported the beneficial role of Zhi Gan prescription against hepatic steatosis and liver injury, which are the same hallmarks of ALD (Qin et al.). It is worth looking into this anti-ALD effect in a future study.

\section{CONCLUSION}

In summary, this special issue contains eight representative articles, including seven original articles and one review. Evidence from these articles has greatly promoted our scientific understanding of the pathological mechanisms and biological prevention and treatment of ALD. This topic has so far attracted wide attention with over 12,700 views.

\section{AUTHOR CONTRIBUTIONS}

All authors listed have made a substantial, direct, and intellectual contribution to the work and approved it for publication.

Dou, X., Feng, L., Ying, N., Ding, Q., Song, Q., Jiang, F., et al. (2020). RNA Sequencing Reveals a Comprehensive Circular RNA Expression Profile in a Mouse Model of Alcoholic Liver Disease. Alcohol. Clin. Exp. Res. 44 (2), 415-422. doi:10.1111/acer.14265

Dou, X., Yang, W., Ding, Q., Han, Q., Qian, Q., Du, Z., et al. (2021). Comprehensive Analysis of the Expression Profiles of Hepatic lncRNAs in the Mouse Model of Alcoholic Liver Disease. Front. Pharmacol. 12, 709287. doi:10.3389/fphar.2021. 709287

GBD 2016 Alcohol Collaborators (2018). Alcohol Use and burden for 195 Countries and Territories, 1990-2016: a Systematic Analysis for the Global Burden of Disease Study 2016. Lancet 392 (10152), 1015-1035. doi:10.1016/S0140-6736(18)31310-2

Hartmann, P., Seebauer, C. T., and Schnabl, B. (2015). Alcoholic Liver Disease: the Gut Microbiome and Liver Cross Talk. Alcohol. Clin. Exp. Res. 39 (5), 763-775. doi:10.1111/acer.12704 
Hritz, I., Mandrekar, P., Velayudham, A., Catalano, D., Dolganiuc, A., Kodys, K., et al. (2008). The Critical Role of Toll-Like Receptor (TLR) 4 in Alcoholic Liver Disease is Independent of the Common TLR Adapter MyD88. Hepatology 48 (4), 1224-1231. doi:10.1002/hep.22470

Patterson, E., Wall, R., Fitzgerald, G. F., Ross, R. P., and Stanton, C. (2012). Health Implications of High Dietary omega-6 Polyunsaturated Fatty Acids. J. Nutr. Metab. 2012, 539426. doi:10.1155/2012/539426

Seitz, H. K., Bataller, R., Cortez-Pinto, H., Gao, B., Gual, A., Lackner, C., et al. (2018). Alcoholic Liver Disease. Nat. Rev. Dis. Primers 4 (1), 16. doi:10.1038/ s41572-018-0014-7

Smathers, R. L., Galligan, J. J., Stewart, B. J., and Petersen, D. R. (2011). Overview of Lipid Peroxidation Products and Hepatic Protein Modification in Alcoholic Liver Disease. Chem. Biol. Interact 192 (1-2), 107-112. doi:10.1016/j.cbi.2011. 02.021

Tull, S. P., Yates, C. M., Maskrey, B. H., O’Donnell, V. B., Madden, J., Grimble, R. F., et al. (2009). Omega-3 Fatty Acids and Inflammation: Novel Interactions Reveal a New Step in Neutrophil Recruitment. PLoS Biol. 7 (8), e1000177. doi:10.1371/journal.pbio.1000177

Zhang, Y., Zeng, F., Zeng, M., Han, X., Cai, L., Zhang, J., et al. (2021). Identification and Characterization of Alcohol-Related Hepatocellular
Carcinoma Prognostic Subtypes Based on an Integrative N6Methyladenosine Methylation Model. Int. J. Biol. Sci. 17 (13), 3554-3572. doi:10.7150/ijbs.62168

Conflict of Interest: The authors declare that the research was conducted in the absence of any commercial or financial relationships that could be construed as a potential conflict of interest.

Publisher's Note: All claims expressed in this article are solely those of the authors and do not necessarily represent those of their affiliated organizations, or those of the publisher, the editors and the reviewers. Any product that may be evaluated in this article, or claim that may be made by its manufacturer, is not guaranteed or endorsed by the publisher.

Copyright (C) $2022 \mathrm{Li}$, Song, Yao and Qin. This is an open-access article distributed under the terms of the Creative Commons Attribution License (CC BY). The use, distribution or reproduction in other forums is permitted, provided the original author(s) and the copyright owner(s) are credited and that the original publication in this journal is cited, in accordance with accepted academic practice. No use, distribution or reproduction is permitted which does not comply with these terms. 